This item was submitted to Loughborough's Research Repository by the author.

Items in Figshare are protected by copyright, with all rights reserved, unless otherwise indicated.

\title{
Microscopic theory for the Markovian decay of magnetization fluctuations in nanomagnets
}

\section{PLEASE CITE THE PUBLISHED VERSION}

https://doi.org/10.1103/PhysRevB.76.214431

\section{PUBLISHER}

(C) American Physical Society (APS)

\section{VERSION}

AM (Accepted Manuscript)

\section{PUBLISHER STATEMENT}

This work is made available according to the conditions of the Creative Commons Attribution-NonCommercialNoDerivatives 4.0 International (CC BY-NC-ND 4.0) licence. Full details of this licence are available at: https://creativecommons.org/licenses/by-nc-nd/4.0/

\section{LICENCE}

CC BY-NC-ND 4.0

\section{REPOSITORY RECORD}

Rousochatzakis, loannis. 2019. "Microscopic Theory for the Markovian Decay of Magnetization Fluctuations in Nanomagnets". figshare. https://hdl.handle.net/2134/34418. 


\title{
Microscopic Theory for the Markovian Decay of Magnetization Fluctuations in Nanomagnets
}

\author{
Ioannis Rousochatzaki.* \\ Institut de théorie des phénomènes physiques, Ecole Polytechnique Fédérale de Lausanne, \\ CTMC, BSP - Dorigny, CH-1015 Lausanne, Switzerland
}

\begin{abstract}
We present a microscopic theory for the phonon-driven decay of the magnetization fluctuations in a wide class of nanomagnets where the dominant energy is set by isotropic exchange and/or uniaxial anisotropy. Based on the Zwanzig-Mori projection formalism, the theory reveals that the magnetization fluctuations are governed by a single decay rate $\omega_{c}$, which we further identify with the zero-frequency portion of the associated self-energy. This dynamical decoupling from the remaining slow degrees of freedom is attributed to a conservation law and the discreteness of the energy spectrum, and explains the omnipresent mono-exponential decay of the magnetization over several decades in time, as observed experimentally. A physically transparent analytical expression for $\omega_{c}$ is derived which highlights the three specific mechanisms of the slowing down effect which are known so far in nanomagnets.
\end{abstract}

PACS numbers: 75.50.Xx, 75.75.+a, 76.60.Es

\section{INTRODUCTION}

Over the past two decades there has been an intense theoretical and experimental interest in the field of molecular nanomagnets 1,2 in view of their importance in the general context of quantum magnetism but also for potential applications, e.g. in quantum computing ${ }^{3.4}$, memory storage $e^{\frac{5}{}}$, and magnetic imaging ${ }^{6}$. The dominant energy in these "zero-dimensional" magnets is set by intramolecular isotropic exchange interactions and/or easyaxis anisotropy. Two of the most central motifs in this research field have been ${ }^{2}$ the fact that the decay of the equilibrium fluctuations $J(t)$ of the total magnetization $S_{z}$ is characterized by a single correlation frequency parameter $\omega_{c}$ (or inverse decay time $1 / \tau_{c}$ ) over several decades in time, and the experimental manifestation of a dramatic slowing down of $\omega_{c}$ which establishes already from relatively high temperatures $T$.

The correlation frequency parameter is a quantity of broad experimental relevance and of great theoretical value. For instance, according to linear response theory and the fluctuation-dissipation theorem, $\omega_{c}$ is probed by ac (longitudinal) susceptibility and magnetization relaxation measurements. It may also be measured directly by Electron Spin Resonance ${ }^{7}$ since $\omega_{c}$ is the electronic spin-lattice relaxation rate $\left(1 / T_{1}\right)_{\mathrm{el}}$. Moreover, if $\omega_{c}$ is in the regime of the nuclear Larmor frequency $\omega_{L}$, then it can be indirectly probed by Nuclear Magnetic Resonance (NMR) as in Antiferromagnetic Rings $8,9,10$. Similarly, $\omega_{c}$ is also of relevance in Muon ${ }^{11}$ and Mössbauer ${ }^{12}$ spectroscopies. Previous theoretical studies of $\omega_{c}^{2,13,14,15,16}$ are based on the Master Equation (ME) which embodies the coupled dynamics of the full set of populations $p_{l}$ of the various eigenstates $|l\rangle$ of the isolated spin Hamiltonian $\mathcal{H}_{s}$. In this approach, one first evaluates the various phonon-driven quantum-mechanical transition rates $W_{l l^{\prime}}$ from $|l\rangle$ to $\left|l^{\prime}\right\rangle$ and then forms and diagonalizes numerically the associated relaxation matrix $\mathcal{R}$. This procedure gives a multi-exponential decay. To account for the observed behavior it is then sometimes argued $2,14,15,16$ that $\omega_{c}$ can be assigned to the lowest eigenvalue (or decay rate) $\lambda_{0}$ of $\mathcal{R}$. Despite being physically quite plausible, such a hypothesis alone does not guarantee that $\lambda_{0}$ corresponds to the decay of the observable of interest (here $S_{z}$ ), since other observables (such as the magnetic energy $\mathcal{H}_{s}$ ) decay slowly as well. As shown by Santini et al $\frac{9}{\underline{9}}$, a full numerical treatment of this problem based on the ME approach requires also the calculation of the weight of each eigenvalue of $\mathcal{R}$ in $J(t)$. In agreement with experimental data, the ME calculations of Santini et $a l^{9} \stackrel{9}{ }$ showed that $J(t)$ is dominated by a single eigenvalue and which, indeed, does not always equal $\lambda_{0}$. A principal theoretical question however still remains as to whether or not there is a physical reason which necessitates the observed dynamical decoupling of $S_{z}$ over several decades in time and in a large class of magnetic molecule clusters.

In this article we put forward a theoretical framework which focuses right from the start on the principal quantity of interest, namely the fluctuations $J(t)$ of the total magnetization $S_{z}$. This approach reveals immediately the physical origin of the observed Markovian decay: The self-energy $\Sigma(t)$ of the magnetization embodies fluctuations of non-conserved observables which decay much faster than $S_{z}$. It is shown that this separation of time scales is guaranteed by the discreteness of the magnetic energy spectrum. The identification of the precise eigenvalue of $\mathcal{R}$ which governs the decay of $J(t)$ with the zerofrequency portion of the self-energy and the derivation of an analytical expression for it (cf. Eq. (27) or (31)) in lowest order in the magnetoelastic coupling is a second achievement of this approach. This expression is an optimal starting point for direct and quantitative comparison with experimental data and thus allows to explore the specific low-energy phonon modes which are involved in the magnetoelastic process in all nanomagnets, an issue which is of broad interest and is currently largely unexplored. At the same time, this expression readily 
demonstrates the three specific mechanisms of the slowing down effect which are known so far in nanomagnets: (i) the existence of an easy-axis anisotropy, (ii) the increasingly gapped character of the spectrum at low energies, and (iii) the possibility of a crossover to a critical slowing down.

The first is present in Single Molecule Magnets (SMM's) ${ }^{2,17,18}$ which are perhaps the most intensively studied nanomagnets since, apart from the above "superparamagnetic" behavior, they also provide a remarkable manifestation of magnetization tunneling ${ }^{19}$. Here, the presence of competing exchange interactions stabilizes a ferrimagnetic ground state of a large total spin $S$, e.g. $S=10$ for the $\mathrm{Mn}_{12}$ and $\mathrm{Fe}_{8}$ clusters. The energy scale within this large $S$ manifold is set by an effective uniaxial anisotropy $-|D| S_{z}^{2}$, giving rise to a correspondingly large barrier $\left(U \simeq 62 \mathrm{~K} \text { for } \mathrm{Mn}_{12} \text { and } 24.5 \mathrm{~K} \text { for } \mathrm{Fe}_{8}\right)^{2,17,18}$ for thermal relaxation. The relaxation time $\tau_{c}$ of $S_{z}$ follows a phenomenological Arrhenius law $\tau_{c} \propto e^{U / k_{B} T}$ which can reach values as long as $10^{5}-10^{7}$ sec at low $T$. This behavior is also revealed by complementary ac susceptibility measurements ${ }^{2}$.

The second mechanism appears e.g. in Antiferromagnetic Rings AFMR's. These are planar ring-like magnetic clusters comprising an even number $N$ of spins $s$ whereby the dominant energy is set by the nearest-neighbor antiferromagnetic (AFM) exchange $J$. This stabilizes a nonmagnetic $S=0$ ground state separated by a spin gap $\Delta \simeq 4 J / N$ (Landé interval rule) from the lowest $S=1$ excitation. Here there is no energy barrier and thus the corresponding $\omega_{c}$ are on a much faster scale than the ones in SMM's. Still, it has been found $8,9,10$ that $\omega_{c}$ drops by several (three up to five) decades as $T$ drops below $\Delta / k_{B}$. This dramatic decrease is systematically revealed by an enhancement of the nuclear spin-lattice relaxation rate $1 / T_{1}$ at $T \sim \Delta / k_{B}{ }^{8,9,10}$. A similar behavior of $1 / T_{1}$ is observed in other AFM clusters but also in SMM's20. In the latter, the analysis of $1 / T_{1}$ is hindered by the NMR wipe-out effect ${ }^{20}$.

The third mechanism of the slowing down effect emerges in Single Chain Magnets (SCM's) 21,22,23,24,25 which comprise finite ferromagnetic chains of variant lengths $L$. Here, a crossover from an activated to a critical slowing down occurs as we tend toward the $T=0$ (thermodynamically) critical point, and in particular when the correlation length $\xi$ approaches $L$. Our approach accommodates such a mechanism exactly as in critical bulk systems ${ }^{26}$ since it reveals that $\omega_{c}$ is inversely proportional to the isothermal magnetic susceptibility $\chi_{0}$ (cf. Eq. (27)).

The remainder of this article is organized as follows. In Sec. II we describe the main terms of the spin Hamiltonian of the magnetic cluster and its interaction with the host lattice which drives the relaxational dynamics. In Sec. III A we introduce the so-called Mori correlation function and express the main quantities of interest in terms of it. The Zwanzig-Mori projection technique is then employed in Sec. IIIB in order to intro- duce the self-energy function and highlight its major role. A perturbative expansion of this quantity is then given up to lowest (second) order in the magnetoelastic coupling. In Sec. IIC we provide a rigorous justification of the Markov approximation based on the discreteness of the magnetic energy spectrum. We then arrive at our expression for the correlation frequency and provide a qualitative analysis of the three specific mechanisms of the slowing-down effect which are known so far in nanomagnets. In Sec. IV we revisit the problem of nuclear spin-lattice relaxation in AFMR's and clarify a number of aspects in the light of our treatment. Finally we give a short summary and overview of our results in Sec. V.

\section{HAMILTONIAN}

At not too low $T$, and given the typical energy scale of the magnetic excitations (a few tens of degrees Kelvin), the decay of the electronic spin fluctuations must originate from the coupling to the deformations of the host lattice, and in particular, the low-energy phonon modes. The Hamiltonian of the full spin+phonon system can be generally written as

$$
\mathcal{H}=\mathcal{H}_{s}+\mathcal{H}_{p h}+\mathcal{V}
$$

where $\mathcal{H}_{s}$ stands for the isolated spin Hamiltonian, $\mathcal{H}_{p h}$ is the phonon energy and $\mathcal{V}$ denotes the relevant spinphonon interaction channel(s). The first is taken to include the most dominant magnetic energy terms

$$
\mathcal{H}_{s}=\sum_{i j} J_{i j} \mathbf{s}_{i} \cdot \mathbf{s}_{j}-|D| S_{z}^{2}+g \mu_{B} B S_{z},
$$

i.e., the isotropic Heisenberg exchange interactions (leading term), a possible effective easy-axis anisotropy (second term), and finally a Zeeman term for the coupling between an external longitudinal (along the easy $z$-axis) field $\mathbf{B}$ and the total magnetic moment $S_{z}=\sum_{i} s_{i}^{z}$. As usually, $g$ denotes the electronic spectroscopic factor and $\mu_{B}$ is the Bohr magneton. In what follows, we shall denote by $|l\rangle$ and $E_{l}$ the eigenstates and eigenvalues of $\mathcal{H}_{s}$ and by $M_{l}$ the corresponding eigenvalues of $S_{z}$. In our treatment we have excluded Dzyaloshinskii-Moriya interactions and transverse on-site anisotropies which are very small $(\lesssim 1 \mathrm{~K})$ and are relevant at ultra low temperatures only. As we show below, the observed mono-exponential decay of $S_{z}$ can be explained by the most dominant energy terms which are included in Eq. (2).

For the magnetoelastic coupling $\mathcal{V}$, we may write without loss of generality

$$
\mathcal{V}=\sum_{\mu} \mathcal{A}^{\mu} \otimes \mathcal{B}^{\mu}
$$

where $\mathcal{A}^{\mu}$ and $\mathcal{B}^{\mu}$ are spin and phonon operators respectively. The latter represent low-energy strain fields probed by the electronic spin degrees of freedom. Details 
about the origin and possible types of $\mathcal{V}$ are discussed e.g. in Refs. 2, 13, 27, 28,29. As we show below, the major phonon quantities that govern the dissipative dynamics of the electronic spin fluctuations are the spectral densities

$$
J_{\mu \mu^{\prime}}^{p h}(\omega) \equiv \int_{-\infty}^{\infty} d t e^{i \omega t}\left\langle\mathcal{B}^{\mu} \mathcal{B}^{\mu^{\prime}}(t)\right\rangle_{\mathrm{ph}}
$$

where the thermal average is taken over the canonical ensemble $\rho_{p h}(T)=e^{-\beta \mathcal{H}_{p h}} / Z_{p h}, Z_{p h}=\operatorname{Tr} e^{-\beta \mathcal{H}_{p h}}$.

\section{MAGNETIZATION FLUCTUATIONS}

In the absence of spin-phonon coupling, $S_{z}$ is conserved since $\left[S_{z}, \mathcal{H}_{s}\right]=0$. If the observables coupled to $S_{z}$ (through $\mathcal{V}$ ) do not share this property it is physically expected that they decay faster than $S_{z}$. This separation of time scales is at the origin of the Markovian decay of $S_{z}$. And such a central physical ingredient can be exploited naturally within the framework of Zwanzig-Mori projection formalism $26,30,31,32,33,34$, whereby one may isolate the important slow degrees of freedom by appropriately projecting out the remaining faster ones. To this end, it is expedient to first express the central quantities of interest in terms of the so-called Mori correlation function which can be readily handled with the Zwanzig-Mori projection technique.

\section{A. Quantities of central interest in terms of the Mori correlation function}

Let us first introduce the Mori correlation function. We first note that any operator $A$ can be represented as a "state vector" $\mid A$ ) in the Liouville space (space of operators). A convenient choice of a scalar product in this vector space is the so-called Mori product defined as

$$
(A \mid B) \equiv \frac{1}{\beta} \int_{0}^{\beta} d x\left\langle A^{\dagger} B(i \hbar x)\right\rangle=\left\langle A^{\dagger} \frac{1-e^{-\beta \hbar \mathcal{L}}}{\beta \hbar \mathcal{L}} B\right\rangle,
$$

where $B(i \hbar x) \equiv e^{-\hbar \mathcal{L} x} B=e^{-x \mathcal{H}} B e^{x \mathcal{H}}$, and $\mathcal{L}=\mathcal{L}_{s}+$ $\mathcal{L}_{p h}+\mathcal{L}_{\mathcal{V}} \equiv \mathcal{L}_{0}+\mathcal{L}_{\mathcal{V}}$ denotes the Liouville operator. Its action on a given operator $A$ is defined as

$$
i \mathcal{L} A \equiv \frac{1}{i \hbar}[A, \mathcal{H}]=\left.\frac{d A(t)}{d t}\right|_{t=0} \equiv \dot{A}
$$

Also, the thermal average is taken over the canonical ensemble $\rho(T)$ of the full spin + phonon system

$$
\rho(T)=Z^{-1} e^{-\beta \mathcal{H}}, Z=\operatorname{Tr} e^{-\beta \mathcal{H}}
$$

where $\beta=1 /\left(k_{B} T\right)$ denotes the inverse temperature and $k_{B}$ is the Bolzmann's constant. In what follows we assume that $\beta \mathcal{V} \ll 1$ and thus the magnetoelastic coupling cannot influence static properties, i.e. the canonical density matrix factorizes as

$$
\rho(T) \simeq \rho_{s}(T) \otimes \rho_{p h}(T)
$$

where $\rho_{p h}(T)$ was defined previously while $\rho_{s}(T)=$ $e^{-\beta \mathcal{H}_{s}} / Z_{s}, Z_{s}=\operatorname{Tr} e^{-\beta \mathcal{H}_{s}}$. Now, the Mori correlation function $M(t)$ is nothing else but the projection of the time-evolved "state" $\left.\left.\mid \delta S_{z}(t)\right)=e^{i \mathcal{L} t} \mid \delta S_{z}\right)$ onto $\left.\mid \delta S_{z}\right)$, i.e.

$$
M(t) \equiv\left(\delta S_{z} \mid \delta S_{z}(t)\right)=\left\langle\delta S_{z} \frac{1-e^{-\beta \hbar \mathcal{L}}}{\beta \hbar \mathcal{L}} \delta S_{z}(t)\right\rangle,
$$

where $\delta S_{z} \equiv S_{z}-\left\langle S_{z}\right\rangle$ and $\delta S_{z}(t) \equiv e^{i \mathcal{H} t / \hbar} \delta S_{z} e^{-i \mathcal{H} t / \hbar}=$ $e^{i \mathcal{L} t} \delta S_{z}$. The one-sided Fourier (or Laplace) transform of $M(t)$ reads

$$
\begin{aligned}
\mathcal{M}(s) & \equiv\left(\delta S_{z} \mid \delta S_{z}\right)_{s}=\int_{0}^{\infty} d t e^{i s t}\left(\delta S_{z} \mid e^{i \mathcal{L} t} \delta S_{z}\right) \\
& =\left(\delta S_{z} \mid \frac{i}{s+\mathcal{L}} \delta S_{z}\right)
\end{aligned}
$$

On the other hand, the experimentally most relevant quantities which embody the dynamics of the magnetization fluctuations are the spectral density $\mathcal{J}(\omega)$ and the dynamical susceptibility $\chi(s)$. The first is the Fourier transform of the product correlation function $J(t)$, i.e.

$$
\mathcal{J}(\omega) \equiv \int_{-\infty}^{\infty} d t e^{i \omega t}\left\langle\delta S_{z} \delta S_{z}(t)\right\rangle
$$

while the second is the one-sided Fourier transform of the commutator correlation function

$$
\chi(s) \equiv \int_{0}^{\infty} d t e^{i s t}\left\langle\frac{1}{i \hbar}\left[\delta S_{z}, \delta S_{z}(t)\right]\right\rangle, s=\omega+i \eta, \eta>0 .
$$

The two quantities are connected via the fluctuation dissipation theorem

$$
\mathcal{J}(\omega)=\frac{2 \hbar}{1-e^{-\beta \hbar \omega}} \chi^{\prime \prime}\left(\omega+i 0^{+}\right) .
$$

It is now straightforward to express the dynamical susceptibility $\chi(s)$ in terms of $\mathcal{M}(s)$ using their definitions Eqs. (10) and (12), and the Kubo identity $\beta(A \mid \dot{B})=$ $-\left\langle\frac{1}{i \hbar}\left[A^{\dagger}, B\right]\right\rangle$ which follows from Eq. (5) by replacing $B$ with $\dot{B}$ and using the relation $\left\langle A^{\dagger} e^{-\beta \hbar \mathcal{L}} B\right\rangle=\left\langle B A^{\dagger}\right\rangle$. The resulting expression is

$$
\begin{aligned}
\chi(s) & =-\beta\left(\delta S_{z} \mid \delta \dot{S}_{z}\right)_{s}=\beta\left(\delta S_{z} \mid \frac{\mathcal{L}}{s+\mathcal{L}} \delta S_{z}\right) \\
& =\chi_{0}+i \beta s \mathcal{M}(s),
\end{aligned}
$$

where $\chi_{0}$ is the isothermal differential susceptibility:

$$
\begin{aligned}
\chi_{0} & \equiv \beta\left(\delta S_{z} \mid \delta S_{z}\right)=\beta\left\langle\delta S_{z} \frac{1-e^{-\beta \hbar \mathcal{L}}}{\beta \hbar \mathcal{L}} \delta S_{z}\right\rangle \\
& =\beta\left\langle\left(\delta S_{z}\right)^{2}\right\rangle=-\frac{1}{g \mu_{B}} \frac{\partial\left\langle S_{z}\right\rangle}{\partial B} .
\end{aligned}
$$

The second line follows from $\beta \mathcal{L} \simeq \beta\left(\mathcal{L}_{s}+\mathcal{L}_{p h}\right)$ (since $\beta \mathcal{V} \ll 1)$ and $\hbar L_{s} S_{z}=\left[\mathcal{H}_{s}, S_{z}\right]=0$. 


\section{B. Projection method and self-energy $\Sigma(s)$}

Having at hand Eqs. (14) and (13), we may now concentrate on the Mori correlation function which can be easily handled within the Zwanzig-Mori formalism. The idea is to project out from Eq. (10) fluctuations that couple to $S_{z}$ through the resolvent $\mathcal{G}=i(s+\mathcal{L})^{-1}$. This can be done by employing the projection operators

$$
\mathcal{P}=\frac{\left.\mid \delta S_{z}\right)\left(\delta S_{z} \mid\right.}{\left(\delta S_{z} \mid \delta S_{z}\right)}, \mathcal{Q}=1-\mathcal{P}
$$

and then by decomposing $\mathcal{G}$ using the operator identity $(\mathcal{C}+\mathcal{D})^{-1}=\mathcal{C}^{-1}-(\mathcal{C}+\mathcal{D})^{-1} \mathcal{D} \mathcal{C}^{-1}$, with $\mathcal{C}=s+\mathcal{Q} \mathcal{L}$, and $\mathcal{D}=\mathcal{P} \mathcal{L}$. After some straightforward steps we obtain

$$
\mathcal{M}(s)=\frac{\chi_{0}}{\beta} \frac{i}{s+\Omega+i \Sigma(s)}
$$

where we have used $M(t=0)=\left(\delta S_{z} \mid \delta S_{z}\right)=\chi_{0} / \beta$, and introduced the eigenfrequency $\Omega$ and the self-energy or memory function $\Sigma(s)$ which are defined by

$$
\begin{aligned}
i \Omega & =\frac{\beta}{\chi_{0}}\left(\delta S_{z} \mid i \mathcal{L} \delta S_{z}\right)=\frac{\beta}{\chi_{0}}\left(S_{z} \mid \dot{S}_{z}\right) \\
\Sigma(s) & =\frac{\beta}{\chi_{0}}\left(\delta S_{z}\left|\mathcal{L} \mathcal{Q} \frac{i}{s+\mathcal{Q} \mathcal{L} \mathcal{Q}} \mathcal{Q} \mathcal{L}\right| \delta S_{z}\right) .
\end{aligned}
$$

The physical meaning of these quantities becomes transparent by taking the inverse Laplace transform of Eq. (17) which reads

$$
\frac{d}{d t} M(t)=i \Omega M(t)-\int_{0}^{t} d t^{\prime} \Sigma\left(t^{\prime}\right) M\left(t-t^{\prime}\right), t \geq 0 .
$$

This justifies the origin of the terms eigenfrequency and memory function for $\Omega$ and $\Sigma$ respectively.

Equations (17), (18) and (19) are exact. All information about the remaining fluctuations coupled to $S_{z}$ has been conveniently embedded into $\Sigma(s)$. Here $\Omega=0$ due to the definite time reversal signature of $S_{z}$ (also due to the Kubo identity given above). Importantly, this also implies that $\left.\left.\left.\mathcal{Q} \mid \dot{S}_{z}\right)=(1-\mathcal{P}) \mid \dot{S}_{z}\right)=\mid \dot{S}_{z}\right)$, which simplifies Eq. (19) to

$$
\Sigma(s)=\frac{\beta}{\chi_{0}}\left(\dot{S}_{z}\left|\frac{i}{s+\mathcal{L}}\right| \dot{S}_{z}\right)=\frac{\beta}{\chi_{0}}\left(\dot{S}_{z} \mid \dot{S}_{z}\right)_{s} .
$$

Hence, $\Sigma\left(\omega+i 0^{+}\right)$is proportional to the Mori correlation function $\left(\dot{S}_{z} \mid \dot{S}_{z}\right)_{\omega+i 0^{+}}$and thus embodies the fluctuations of the "flux" operator 37

$$
\dot{S}_{z}=\frac{1}{i \hbar}\left[S_{z}, \mathcal{V}\right]=\sum_{\mu} \mathcal{F}^{\mu} \otimes \mathcal{B}^{\mu}
$$

where $\mathcal{F}^{\mu} \equiv \frac{1}{i \hbar}\left[S_{z}, \mathcal{A}^{\mu}\right]$. For later convenience let us write the matrix elements of these operators: $\mathcal{F}_{l l^{\prime}}^{\mu} \equiv$ $\left\langle l\left|\mathcal{F}^{\mu}\right| l^{\prime}\right\rangle=\frac{i}{\hbar} \delta M_{l^{\prime} l} \mathcal{A}_{l l^{\prime}}^{\mu}$, where $\delta M_{l^{\prime} l} \equiv M_{l^{\prime}}-M_{l}$.
We now employ a perturbation expansion for the evaluation of the self-energy $\Sigma\left(\omega+i 0^{+}\right)$. To this end, one needs to expand the resolvent $\mathcal{G}=i(s+\mathcal{L})^{-1}$ of Eq. 21 in powers of $\mathcal{L} \mathcal{V}$. Interestingly, $\dot{S}_{z}$ is linear in $\mathcal{V}$ and thus we may already obtain the lowest order effect by simply replacing $\mathcal{L}$ by $\mathcal{L}_{0}=\mathcal{L}_{s}+\mathcal{L}_{p h}$ in the denominator of Eq. (17). To second order then, $\left(\dot{S}_{z} \mid \dot{S}_{z}\right)_{\omega+i 0^{+}}$equals to

$$
\begin{array}{r}
\left(\dot{S}_{z}\left|\frac{i}{\mathcal{L}_{0}+\omega+}+i 0^{+}\right| \dot{S}_{z}\right)=\left(\dot{S}_{z}\left|\pi \delta\left(\mathcal{L}_{0}+\omega\right)\right| \dot{S}_{z}\right) \\
=\pi \frac{e^{\beta \hbar \omega}-1}{\beta \hbar \omega}\left\langle\dot{S}_{z} \delta\left(\mathcal{L}_{0}+\omega\right) \dot{S}_{z}\right\rangle
\end{array}
$$

where we have used the reality of $\Sigma\left(\omega+i 0^{+}\right)$. Employing the representation $\delta\left(\mathcal{L}_{0}+\omega\right)=(2 \pi)^{-1} \int d t e^{i \mathcal{L}_{0} t}$, factorizing $e^{i \mathcal{L}_{0} t}=e^{i \mathcal{L}_{s} t} e^{i \mathcal{L}_{p h} t}$, and using Eq. (8) we obtain

$\Sigma\left(\omega+i 0^{+}\right)=\frac{\beta}{\chi_{0}} \frac{e^{\beta \hbar \omega}-1}{2 \beta \hbar \omega} \sum_{\mu \mu^{\prime}} \int d \omega^{\prime} J_{\mu \mu^{\prime}}^{s}\left(\omega^{\prime}\right) J_{\mu \mu^{\prime}}^{p h}\left(\omega-\omega^{\prime}\right)$,

where $J_{\mu \mu^{\prime}}^{p h}(\omega)$ was defined previously, while $J_{\mu \mu^{\prime}}^{s}(\omega)$ stand for the isolated spectral densities of the $\mathcal{F}^{\mu}$ operators, i.e.

$$
J_{\mu \mu^{\prime}}^{s}(\omega)=\int_{-\infty}^{\infty} d t e^{i \omega t}\left\langle\mathcal{F}^{\mu} \mathcal{F}^{\mu^{\prime}}(t)\right\rangle_{\mathrm{s}},
$$

where the thermal average is taken over the canonical ensemble $\rho_{\mathrm{S}}(T)$.

\section{Markovian behavior and correlation frequency}

The origin of the mono-exponential decay of $S_{z}$ follows immediately from Eq. (24) and this is related to the discrete character of the spectrum. The spectral densities $J^{s}\left(\omega^{\prime}\right)$ of $\mathcal{F}_{\mu}$ are peaked at the characteristic Bohr frequencies $\omega^{\prime}=\left(E_{l}-E_{l^{\prime}}\right) / \hbar$ of $\mathcal{H}_{s}$. On the other hand, we are interested in a long time regime: For all relevant experimental frequency scales (e.g. $\omega_{L}$ in NMR) $\omega \sim \omega_{c} \ll$ $\omega^{\prime}$, and thus, given the extremely short phonon correlation times, we may replace ${ }^{38} \mathcal{J}^{p h}\left(\omega-\omega^{\prime}\right) \simeq J^{p h}\left(-\omega^{\prime}\right)$, which is equivalent with the usual Markov approximation of replacing $\Sigma\left(\omega+i 0^{+}\right) \simeq \Sigma\left(i 0^{+}\right)$in Eq. (17). This "memory-less" character of the self-energy means that the fluctuations of the observables coupled to $S_{z}$ (i.e. the observables $\mathcal{F}^{\mu}$ that enter in $\dot{S}_{z}$ ) decay in times $\tau_{c}^{\prime}$ much shorter than the decay time $\tau_{c}=1 / \omega_{c}$ of $M(t)$. The physical origin of this separation of time scales can be attributed to the fact that, in contrast to $\mathcal{F}^{\mu}, S_{z}$ is a conserved variable for the isolated magnetic cluster and thus is expected to decay much slower in the presence of the small magnetoelastic coupling. At the same time, this separation of time scales is at the origin of the Markovian decay of $S_{z}$ : As long as $t \gg \tau_{c}^{\prime}$, one may replace $M\left(t-t^{\prime}\right)$ with $M(t)$ inside the integral of Eq. (20) and extend the upper limit to infinity, i.e. 
$\frac{d}{d t} M(t) \simeq-\int_{0}^{\infty} d t^{\prime} \Sigma\left(t^{\prime}\right) \cdot M(t) \equiv-\Sigma\left(i 0^{+}\right) \cdot M(t)$, which gives 39

$$
M(t)=\left(\chi_{0} / \beta\right) e^{-\Sigma\left(i 0^{+}\right) t}, t \geq 0
$$

We can thus identify the zero-frequency portion of the self-energy with the correlation frequency $\omega_{c}$ :

$$
\omega_{c}=\Sigma\left(i 0^{+}\right)=\frac{\beta}{2 \chi_{0}} \sum_{\mu \mu^{\prime}} \int_{-\infty}^{\infty} d \omega^{\prime} \mathcal{J}_{\mu \mu^{\prime}}^{s}\left(\omega^{\prime}\right) \mathcal{J}_{\mu \mu^{\prime}}^{p h}\left(-\omega^{\prime}\right)
$$

On the other hand, according to Eqs. (14) and (13), the susceptibility and the spectral density have the familiar Debye (or Lorentzian) form:

$$
\begin{aligned}
\chi(s) & =\chi_{0} \frac{i \omega_{c}}{s+i \omega_{c}} \\
\mathcal{J}(\omega) & =\frac{2 \hbar}{1-e^{-\beta \hbar \omega}} \chi_{0} \frac{\omega \omega_{c}}{\omega^{2}+\omega_{c}^{2}} .
\end{aligned}
$$

Equations (27), (28) and (29) are our central results. It is expedient to rewrite Eq. (27) in a more convenient form, in terms of the various quantum-mechanical transition rates $W_{l l^{\prime}}$ from $|l\rangle$ to $\left|l^{\prime}\right\rangle$. To this end, we use the spectral representation $\mathcal{J}_{\mu \mu^{\prime}}^{s}\left(\omega^{\prime}\right)=2 \pi \sum_{l l^{\prime}} P_{l} \mathcal{F}_{l l^{\prime}}^{\mu} \mathcal{F}_{l^{\prime} l}^{\mu^{\prime}} \delta\left(\omega^{\prime}-\omega_{l l^{\prime}}\right)$, where $P_{l} \equiv e^{-\beta E_{l}} / Z_{s}$ and $\mathcal{F}_{l l^{\prime}}^{\mu}$ were given previously. We find

$$
\frac{\omega_{c}}{2 \pi}=\frac{1}{2} \sum_{l, l^{\prime}} P_{l} \frac{\delta M_{l^{\prime} l}^{2}}{\left\langle\delta S_{z}^{2}\right\rangle} \cdot\left[\frac{1}{\hbar^{2}} \sum_{\mu \mu^{\prime}} \mathcal{A}_{l l^{\prime}}^{\mu} \mathcal{A}_{l^{\prime} l}^{\mu^{\prime}} \mathcal{J}_{\mu \mu^{\prime}}^{p h}\left(\omega_{l^{\prime} l}\right)\right]
$$

The last factor of this equation can be identified to be the quantum-mechanical transition rate $W_{l l^{\prime}}$ from $|l\rangle$ to $\left|l^{\prime}\right\rangle$. We therefore obtain the convenient expression

$$
\frac{\omega_{c}}{2 \pi}=\frac{1}{2} \sum_{l, l^{\prime}} P_{l} \frac{\delta M_{l^{\prime} l}^{2}}{\left\langle\delta S_{z}^{2}\right\rangle} W_{l l^{\prime}}
$$

We remark here that we can eliminate the factor of $1 / 2$ appearing in the right hand sides of Eqs. (27) and (31) by restricting the integral of (27) to positive (or negative) frequencies only, or by constraining the sum of (31) to run over $l<l^{\prime}$. This is allowed by the detailed balance condition $\mathcal{J}_{\mu \mu^{\prime}}^{i}\left(-\omega^{\prime}\right)=e^{-\beta \hbar \omega^{\prime}} \mathcal{J}_{\mu^{\prime} \mu}^{i}\left(\omega^{\prime}\right)(i=\mathrm{s}$, ph) or its equivalent form $P_{l} W_{l l^{\prime}}=P_{l^{\prime}} W_{l^{\prime} l}$.

Let us now describe in qualitative terms the main features of Eq. (27) or Eq. (31). According to these expressions, $\omega_{c}$ is a weighted sum over all relevant resonance channels between the spin and the phonon system. Each contribution enters in a transparent and physically appealing factorized form. Energy conservation is ensured by the opposite frequency arguments in $\mathcal{J}^{s}$ and $\mathcal{J}^{\text {ph }}$. The one-phonon portion of the latter is proportional to the number of available (i.e. thermally excited) phonons in resonance with the electronic spin system. We also note that it is $\mathcal{F}_{\mu}$ and $\mathcal{B}_{\mu}$ triggering the decay of $S_{z}$ (cf. (22)) that enter in Eq. (27). This is also reflected in Eq. (31) by the fact that transitions between two levels $l, l^{\prime}$ contribute to $\omega_{c}$ only if they differ in their magnetic moment. More generally, each of the three known mechanisms of the slowing down effect can be now associated to one of the three major quantities appearing in Eq. (27), namely $\mathcal{J}^{p h}, \mathcal{J}^{s}$ and $\chi_{0}$. In AFMR's (see also Sec. IV below), it is the drastic reduction of the resonant portions of the phonon spectral weight $\mathcal{J}^{p h}$ as $T$ drops below the spin gap. In SMM's on the other hand, the effect appears already well above the typical intra-barrier excitations, and thus must be attributed to selection rules in $\mathcal{J}^{s}$ which necessitate an "over the barrier" relaxation process. Finally, the appearance of the susceptibility in the denominator of Eq. (27) is underlying the critical slowing down observed in SCM's $21,22,23,24,25$ similarly to bulk ferromagnets 26 .

We should finally note here that one may start with the magnetic energy $\mathcal{H}_{s}$ instead of $S_{z}$ and show, following exactly the same steps as above, that this observable decays also independently from the remaining slow degrees of freedom. Its corresponding decay rate $\omega_{E}$ can be also derived and the result is fully analogous to Eq. (31):

$$
\frac{\omega_{E}}{2 \pi}=\frac{1}{2} \sum_{l, l^{\prime}} P_{l} \frac{\delta E_{l^{\prime} l}^{2}}{\left\langle\delta \mathcal{H}_{s}^{2}\right\rangle} W_{l l^{\prime}}
$$

where $\delta E_{l^{\prime} l} \equiv E_{l^{\prime}}-E_{l},\left\langle\delta \mathcal{H}_{s}^{2}\right\rangle=k_{B} T^{2} C_{m}$, and $C_{m}$ is the magnetic specific heat. The "magnetic energy correlation frequency" $\omega_{E}$ is of experimental relevance in time-dependent specific heat measurements 35 and is distinct from $\omega_{c}$. It is nevertheless expected, based on the above discussion, that $\omega_{E}$ also manifests a dramatic slowing down similar to $\omega_{c}$.

\section{NUCLEAR SPIN-LATTICE RELAXATION RATE IN AFMR'S}

Here we revisit the problem of proton $1 / T_{1}$ in AFMR's and clarify a number of aspects in the light of Eqs. (29) and (27) or (31). Quite generally, since the proton spin is a local probe, $1 / T_{1}$ samples all (auto- and pair-) electronic spin spectral densities evaluated at $\omega_{L}$. Remarkably however, in clusters with equivalent ionic spins (such as AFMR's) symmetry arguments together with the fact that $\omega_{L}$ is much smaller than the typical Bohr frequencies $\omega_{B}$ assert that away from level crossings the relevant terms contributing to $1 / T_{1}$ are all proportional to the spectral density of the total magnetization 9.36 , i.e.

$$
\frac{1}{T_{1}}=A \mathcal{J}\left(\omega_{L}\right)
$$

where the proportionality constant $A$ embodies the specific details regarding the geometry and the strength of the nuclear-electron hyperfine coupling ${ }^{10}$. The remaining terms of the electronic spin spectral densities are vanishingly small and become relevant only at field-induced level crossings 36 . For symmetric clusters then and using 
Eq. (29) (with $\left.\beta \hbar \omega_{L} \ll 1\right)$ we obtain

$$
\frac{1}{T_{1}}=\left(2 k_{B} A\right) \chi_{0} T \frac{\omega_{c}(T)}{\omega_{L}^{2}+\omega_{c}(T)^{2}},
$$

which is the Lorentzian form used previously ${ }^{8.9}$.

In AFMR's, $\mathcal{J}^{\mathcal{S}}\left(\omega_{B}\right) /\left(\chi_{0} T\right)$ does not change drastically over the whole temperature range of interest. Hence, the observed dramatic decrease of $\omega_{c}$ and the resulting enhancement $\frac{8.9 .10}{}$ of $1 / T_{1}$ when $\omega_{c}(T)=\omega_{L}$ (cf. Eq. (34) ) stems, as mentioned above, from a drastic reduction of the number of available (thermally excited) phonons which are in resonance with the magnetic cluster. This is because on decreasing temperature the relevant low-lying magnetic excitations are increasingly gapped while the frequency peak of $\mathcal{J}^{\text {ph }}(\omega)$ shifts toward lower $\omega$. Eventually the phonon spectral density peak shifts below $\Delta / \hbar$ and one-phonon processes become ineffective in driving the electronic spin fluctuations, thus resulting in a drastic drop of $\omega_{c}$. One can also explain the observed field independence of $\omega_{C} \underline{8,9,10}$ at intermediate temperatures and for $B \ll \Delta /\left(g \mu_{B}\right)$ as follows. The relevant Bohr frequencies (with $\delta M_{l^{\prime} l} \neq 0$ ) in these temperature and field regimes are $\Delta / \hbar \pm \omega_{e} \omega_{e}$ and $2 \omega_{e}$ (here $\omega_{e}$ is the electron Larmor frequency). These frequencies correspond respectively to the transitions between the lowest singlet and the first triplet excitation (with $\delta M_{l^{\prime} l}= \pm \hbar$ ) and to transitions within the lowest triplet. The later do not contribute appreciably since $\mathcal{J}^{p h}\left(\omega_{e}\right) \ll \mathcal{J}^{p h}(\Delta / \hbar)$. At the same time $\mathcal{J}^{p h}\left(\Delta / \hbar \pm \omega_{e}\right) \simeq \mathcal{J}^{p h}(\Delta / \hbar)$ and $\chi_{0}(B) \simeq \chi_{0}(0)$. As a result $\omega_{c}$ is field-independent at intermediate temperatures and $B \ll \Delta /\left(g \mu_{B}\right)$. A field dependence should only arise at higher fields and very low temperatures or for clusters with small $\Delta$.

\section{SUMMARY}

We have presented a microscopic theory for the omnipresent mono-exponential slowing down effect observed in a wide range of molecular nanomagnets. To this end, we first expressed the magnetization fluctuations in terms of the associated self-energy or memory function which embodies the fluctuations of the observables that couple to $S_{z}$. It is then shown that the discreteness of the magnetic energy spectrum guarantees that the self-energy decays in times much shorter than $1 / \omega_{c}$. This separation of time scales results in a dynamical decoupling of the magnetization fluctuations and thus explains their observed Markovian behavior. In addition, we have derived an analytical expression for the correlation frequency parameter $\omega_{c}$ which embodies in a convenient factorized form all essential ingredients. This expression highlights the three specific mechanisms of the slowing down effect which are known so far in nanomagnets, namely (i) the existence of an anisotropy barrier, (ii) the increasingly gapped character of the magnetic energy spectrum at low energies, and (iii) the possibility of a critical slowing down. In addition, this formula contains, in a convenient factorized form, the noise spectra of the relevant low-energy strain fields which are at present largely unexplored. Hence this work paves the way for a more direct and quantitative comparison with experiment and a deeper understanding of the underlying microscopic relaxation channels present in all nanomagnetic systems.

\section{ACKNOWLEDGMENTS}

I would like to thank M. Luban, A. Läuchli, K. P. Schmidt, M. Belesi and F. Mila for very fruitful discussions and valuable comments on the manuscript.
* Electronic address: ioannis.rousochatzakis@epfl.ch

1 O. Kahn, Molecular Magnetism (VCH publishers, New York, 1993).

2 D. Gatteschi, R. Sessoli, and J. Villain, Molecular Nanomagnets (Oxford University Press, Oxford, 2006), and references therein.

3 M. N. Leuenberger and D. Loss, Nature 410, 789 (2001).

4 J. Tejada, E. M. Chudnovsky, E. del Barko, J. M. Hernandez, and T. P. Spiller, Nanotechnology 12, 181 (2001).

${ }^{5}$ G. Christou, D. Gatteschi, D. N. Hendrickson, and R. Sessoli, MRS Bulletin 25, No. 11, 66 (2000).

6 B. Cage, S. E. Russek, R. Shoemaker, A. J. Barker, C. Stoldt, V. Ramachandaran, N. S. Dalal, Polyhedron 26 (2007) 2413-2419.

7 A. Ardavan, O. Rival, J. J. Morton, S. J. Blundell, A. M. Tyryshkin, G. A. Timco, and R. E. Winpenny, Phys.
Rev. Lett 98, 057201 (2007).

8 S. H. Baek, M. Luban, A. Lascialfari, E. Micotti, Y. Furukawa, F. Borsa, J. van Slageren, and A. Cornia, Phys. Rev. B 70, 134434 (2004).

9 P. Santini, S. Carretta, E. Liviotti, G. Amoretti, P. Carretta, M. Filibian, A. Lascialfari, and E. Micotti, Phys. Rev. Lett. 94, 077203 (2005).

10 F. Borsa, A. Lascialfari, Y. Furukawa, NMR in Magnetic Molecular Rings and Clusters, Lect. Notes Phys. 684, 297 (2006), and references therein.

11 L. Cianchi, F. Del Giallo, G. Spina, W. Reiff, and A. Caneschi, Phys. Rev. B 65, 064415 (2002).

12 A. Lascialfari, Z. H. Jang, F. Borsa, P. Carretta, and D. Gatteschi, Phys. Rev. Lett. 81, 3773 (1998).

13 J. Villain, F. Hartman-Boutron, R. Sessoli, and A. Rettori, Europhys. Lett. 27, 301 (1994); F. Hartman-Boutron, 
P. Politi, J. Villain, Int. J. Mod. Phys. B 10, 2577 (1996).

14 M. N. Leuenberger and D. Loss, Europhys. Lett. 46, 692 (1999); Phys. Rev. B 61, 1286 (2000).

15 A. Würger, Phys. Rev. Lett. 81, 212 (1998).

16 L. Bessais, L. Ben Jaffel, and J. L. Dormann, Phys. Rev. B 45, 7805 (1992).

17 R. Sessoli, H. L. Tsai, A. R. Schake, S. Wang, J. B. Vincent, K. Folting, D. Gatteschi, G. Christou, D. N. Hendrickson, J. Am. Chem. Soc. 115,1804 (1993); R. Sessoli, D. Gatteschi, A. Caneschi, M. A. Novak, Nature 365, 141 (1993).

18 C. Sangregorio, T. Ohm, C. Paulsen, R. Sessoli, and D. Gatteschi, Phys. Rev. Lett. 78, 4645 (1997).

19 P. C. E. Stamp, E. M. Chudnovsky, and B. Barbara, Int. J. Mod. Phys. B 6, 1355 (1992); E. M. Chudnovsky and J. Tejada, Macroscopic Quantum Tunneling of the Magnetic Moment (Cambridge University Press, Cambridge, England, 1998).

20 M. Belesi, A. Lascialfari, D. Procissi, Z. H. Jang, and F. Borsa, Phys. Rev. B 72, 014440 (2005); M. Belesi, X. Zong, F. Borsa, C. J. Milios, and S. P. Perlepes, Phys. Rev. B 75, 064414 (2007).

21 A. Canneschi, D. Gatteschi, N. Lalioti, C. Sangregorio, R. Sessoli, G. Venturi, A. Vindigni, A. Rettori, M. G. Pini, M. A. Novak, Angew. Chem., Int. Ed. Engl. 2001, 40, 1760.

22 R. Clérac, H. Miyasaka, M. Yamashita, C. Coulon, J. Am. Chem. Soc. 124, 12837 (2002).

${ }^{23}$ R. Lescouezec, J. Vaissermann, C. Ruiz-Perez, F. Floret, R. Carrasco, M. Julve, M. Verdaguer, Y. Dromzee, D. Gatteschi, and W. Wernsdorfer, Angew. Chem., Int. Ed. Engl. 2003, 42, 1483.

24 C. Coulon, R. Clrac, L. Lecren, W. Wernsdorfer, and H. Miyasaka, Phys. Rev. B 69, 132408 (2004).

${ }^{25}$ H. Miyasaka, R. Clerac, K. Mizushima, K. Sugiura, M. Yamashita, W. Wernsdorfer, and C. Coulon, Inorg. Chem. 42, 8203 (2003).

26 D. Forster, Hydrodynamic Fluctuations, Broken symmetry, and Correlation Functions (W. A. Benjamin, Inc., 1975)

27 E. M. Chudnovsky, D. A. Garanin, and R. Schilling, Phys. Rev. B 72, 094426 (2005).

28 A. Abragam, B. Bleaney, Electron Paramagnetic Resonance of Transition Ions (Clarendon Press, Oxford, 1970).

29 B. Lüthi, in Dynamical Properties of Solids, Vol. 3, edited by G. K. Horton, and A. A. Maradudin (North-Holland Publishing Company, Amsterdam, 1980).

30 H. Mori, Prog. Theor. Phys. 33, 423 (1965).

31 R. Zwanzig, Lectures in Theoretical Physics, Vol. 3 (Interscience, New York, 1961).

32 E. Fick, G. Sauermann, The Quantum Statistics of Dynamic Processes (Springer-Verlag, Berlin, 1990).

33 D. Zubarev, V. Morozov, and G. Röpke, Statistical mechanics of Nonequilibrium processes, Vol. 2 (Akademie Verlag, Berlin, 1997).

34 R. Kubo, M. Toda, and N. Hashitsume, Statistical Physics II, Nonequilibrium Statistical Mechanics (Springer-Verlag, Second Edition, 1991).

35 F. L. Mettes, F. Luis, and L. J. de Jongh, Phys. Rev. B 64, 174411 (2001); M. Evangelisti, F. Luis, F. L. Mettes, R. Sessoli, and L. J. de Jongh, Phys. Rev. Lett. 95, 227206 (2005).

36 I. Rousochatzakis, Ph. D. thesis, Iowa State University (2005).

37 Correlation functions of this type of observables are called generalized Onsager-Casimir coefficients. They play a central role in the entropy production in irreversible processes $32,33,34$.

38 This holds true also for $\omega^{\prime}=0$ given again the extremely short phonon correlation times. Besides, the frequency $\omega_{B}=0$ is absent from the spectral decomposition of $\mathcal{J}^{s}$ since the corresponding matrix elements $\left\langle l\left|\mathcal{F}^{s}\right| l^{\prime}\right\rangle \propto$ $\left\langle l\left|\mathcal{A}^{s}\right| l^{\prime}\right\rangle\left[M_{l^{\prime}}-M_{l}\right]$ vanish for $\hbar \omega_{B}=E_{l}-E_{l^{\prime}}=0$.

39 The same result can be obtained by an inverse Laplace transform of Eq. (17). 